\title{
Cytokine-specific Neurograms in the Sensory Vagus Nerve
}

\author{
Benjamin E Steinberg, ${ }^{1,2^{*}}$ Harold A Silverman, ${ }^{1 *}$ Sergio Robbiati, ${ }^{3 *}$ Manoj K Gunasekaran, ${ }^{1}$ Téa Tsaava, \\ Emily Battinelli, ${ }^{1}$ Andrew Stiegler, ${ }^{4}$ Chad E Bouton, ${ }^{5}$ Sangeeta S Chavan, ${ }^{1+}$ Kevin J Tracey, ${ }^{1+}$ and \\ Patricio T Huerta ${ }^{3,6+}$
}

${ }^{1}$ Laboratory of Biomedical Science, The Feinstein Institute for Medical Research, Northwell Health, Manhasset, New York, United States of America; ${ }^{2}$ Department of Anesthesia, University of Toronto, Toronto, Ontario, Canada; ${ }^{3}$ Laboratory of Immune and Neural Networks, The Feinstein Institute for Medical Research, Northwell Health, Manhasset, New York, United States of America; ${ }^{4}$ Circulatory Technologies, Inc., Manhasset, New York, United States of America; ${ }^{5}$ Center for Bioelectronic Medicine, The Feinstein Institute for Medical Research, Northwell Health, Manhasset, New York, United States of America; and ${ }^{6}$ Department of Molecular Medicine, Hofstra Northwell Health School of Medicine, Manhasset, New York, United States of America

\begin{abstract}
The axons of the sensory, or afferent, vagus nerve transmit action potentials to the central nervous system in response to changes in the body's metabolic and physiological status. Recent advances in identifying neural circuits that regulate immune responses to infection, inflammation and injury have revealed that vagus nerve signals regulate the release of cytokines and other factors produced by macrophages. Here we record compound action potentials in the cervical vagus nerve of adult mice and reveal the specific activity that occurs following administration of the proinflammatory cytokines tumor necrosis factor (TNF) and interleukin $1 \beta$ (IL-1 $\beta$ ). Importantly, the afferent vagus neurograms generated by TNF exposure are abolished in double knockout mice lacking TNF receptors 1 and 2 (TNF-R1/2KO), whereas IL-1 $\beta$-specific neurograms are eliminated in knockout mice lacking IL-1 $\beta$ receptor (IL-1RKO). Conversely, TNF neurograms are preserved in IL-1RKO mice, and IL-1 $\beta$ neurograms are unchanged in TNF-R1/2KO mice. Analysis of the temporal dynamics and power spectral characteristics of afferent vagus neurograms for TNF and IL-1 $\beta$ reveals cytokine-selective signals. The nodose ganglion contains the cell bodies of the sensory neurons whose axons run through the vagus nerve. The nodose neurons express receptors for TNF and IL-1 $\beta$, and we show that exposing them to TNF and IL-1 $\beta$ significantly stimulates their calcium uptake. Together these results indicate that afferent vagus signals in response to cytokines provide a basic model of nervous system sensing of immune responses.
\end{abstract}

Online address: www.bioelecmed.org

doi: 10.15424/bioelectronmed.2016.00007

\section{INTRODUCTION}

Sensory neurons propagate action potentials to the central nervous system (CNS) in response to changes in the chemical, mechanical and electromagnetic environment. This information provides the afferent arc of reflex circuits that maintain homeostasis in the body's cellular metabolism and organ physiology. An emerging field of knowledge has delineated mechanisms for reflex neural circuits that modulate immune responses. A prototypical neural pathway is the inflammatory reflex (1), defined by the electrical signals transmitted in the vagus nerve to the splenic nerve, culminating in a specialized subset of $\mathrm{T}$ lymphocytes that release acetylcholine $(2,3)$. This T cell-derived neurotransmitter signals via a mechanism that depends on $\alpha-7$ nicotinic acetylcholine receptors ( $\alpha-7 \mathrm{nAChR})$ to inhibit cytokine production by splenic macrophages (2).

${ }^{*} B E S, H A S$ and SR should be considered as co-first authors.

${ }^{\dagger}$ SSC, KJT and PTH should be considered as co-senior authors.

Address correspondence to Kevin J Tracey or Patricio T Huerta, Feinstein Institute for Medical Research, Northwell Health, 350 Community Drive, Manhasset, NY 11030 USA. kjtracey@northwell.edu; pato.huerta@gmail.com

Submitted December 8, 2016; Accepted for Publication December 13, 2016;

Published Online December 21,2016 (www. bioelecmed.org).

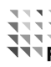

Feinstein Institute
for Medical Research

Northwell Health

The vagus nerve is primarily sensory, and it is the principal conduit that relays afferent signals from the visceral organs to the brainstem. Seminal studies by Linda Watkins revealed that an intact vagus nerve is required for the development of pyrexia in response to intra-abdominal IL-1 $\beta$ administration $(4,5)$. Moreover, early work by Niijima (6-8) suggested that IL-1 $\beta$ might activate signaling in peripheral vagus afferents. Sensory neurons express cytokine receptors, including TNF and IL- $1 \beta$ receptors, and change their activation thresholds when exposed to the corresponding cytokines (9-11). This work, together with other studies, suggests that the vagus nerve may be an important component of a peripheral neural network capable of reporting changes in peripheral inflammation and immunity.

Here we reasoned that the vagus nerve transmits specific neural signatures in response to specific cytokines. 
By analyzing compound action potentials recorded in the cervical vagus nerve that were elicited by exposure of mice to TNF and IL-1 $\beta$, we lay the groundwork for deciphering cytokine-specific vagus nerve signatures.

\section{MATERIALS AND METHODS}

\section{Animals}

All experimental protocols were approved by the Institutional Animal Care and Use Committee at the Feinstein Institute for Medical Research, Northwell Health, which follows National Institutes of Health guidelines for the ethical treatment of animals. We used male BALB/C and C57Bl/6 (8-12 wks, weight 20-30 g), TNFR1/2 double KO (strain B6.129S2Tnfrsf1atm1Imx Tnfrsf1btm1Imx/J, p55- and p75-deficient) and IL-1R KO mice (strain B6.129S7-Il1r1tm1Imx/J), which were purchased from Jackson Laboratory. Animals were housed at $25^{\circ} \mathrm{C}$, with ad libitum water and chow, and acclimated to a $12 \mathrm{~h}$ light and dark cycle for $>3 \mathrm{~d}$ prior to conducting experiments.

\section{Chemicals}

Lidocaine, tetrodotoxin (TTX) (SigmaAldrich) and human IL-1 $\beta$ (eBiosciences) were purchased. Recombinant and tagfree human TNF was produced in-house. Following expression in Escherichia coli, TNF was purified using a cation exchange column and endotoxins removed by phase separation with Triton X-114.

\section{In Vivo Vagus Nerve Recordings}

Mice were fasted for 3-4 h prior to each experiment. They were induced (isoflurane at 2.5\%) and maintained (isoflurane at $\sim 1.75 \%$ ) in the supine position. A midline cervical incision was made and the left cervical branch of the vagus nerve was isolated from the accompanying carotid neurovascular bundle. Under magnification, the connective tissue overlying the nerve was mechanically removed, and the vagus nerve was placed over three custom-built silver hook electrodes or a commercially available bipolar sling cuff electrode with a $200 \mu \mathrm{m}$ inner diameter
(PDMS Sling $\mu$ Cuff, CorTec) (Figure 1). Electrophysiological signals were digitized (sampling rate, $32 \mathrm{kHz}$ ) through a data-acquisition system (Digital Lynx 4SX, Cheetah v5 software, Neuralynx) and referenced to an animal ground electrode placed between the skin and right salivary gland. In all hook electrode experiments, the vagus nerve was protected from desiccation and insulated by bathing the surgical field with mineral oil. Following acquisition of baseline activity (15-20 min), animals were treated with TNF, IL-1 $\beta$ or saline intraperitoneally (i.p.), and recordings were continued for 20-30 min post-injection. The experimenter was grounded whenever manipulating the animal during recordings.

\section{Vagotomies}

A surgical cut of the vagus nerve was completed either proximal or distal to the recording electrodes, using the brain as the point of reference. Following placement of the vagus nerve on the hook electrodes, a silk suture was passed under and secured to the vagus nerve with a single knot. Ethyl cyanoacrylate was used to adhere the vagus nerve to the silk suture and the surgical cut was completed.

\section{Vagus Nerve Evoked Responses}

The vagus nerve was placed on wet tissue paper laid over a custom acrylic platform and kept moist using artificial cerebral spinal fluid (126 mM NaCl, 26 $\mathrm{mM} \mathrm{NaHCO}, 10 \mathrm{mM}$ glucose, $2.5 \mathrm{mM}$ $\mathrm{KCl}, 2.4 \mathrm{mM} \mathrm{CaCl}_{2}, 1.3 \mathrm{mM} \mathrm{MgCl}_{2}$ and $\left.1.2 \mathrm{mM} \mathrm{NaH}_{2} \mathrm{PO}_{4}\right)$. A recording electrode (glass pipette filled with $2 \mathrm{M} \mathrm{NaCl}$ ) and a stimulating electrode (FHC) were placed onto the vagus nerve, with the latter 4-5 mm away from the former (and farther from the head). Signals were amplified ( $\times 1000$, model 1800, AM Systems) and digitized $(30 \mathrm{kHz})$ through an acquisition system (Micro1400 unit and Spike2 version 7 software, CED). Stimuli (20 $\mu$ s long) of increasing intensity (1-50 V) were delivered with a stimulator (SD9, Grass) to generate input/output curves (1 V increments in the 1-10 range, $5 \mathrm{~V}$ increments in the 10-50 range), which were generated first in a saline solution and then in the setting of either lidocaine ( $2 \%)$ or TTX $(100 \mu \mathrm{M})$, a dose inhibitory against voltage-gated sodium channels such as Nav1.8 (14-18). The responses were assessed by integrating the area between the response curve and the baseline value determined 1-10 ms prior to stimulation.

\section{Vagus Nerve Recording Data Analysis}

Spike2 software (version 7, CED) was used for analysis of raw recordings, which were filtered (using high-pass filter with an edge of $160 \mathrm{~Hz}$ ) and smoothed. Neural signals were identified by user-specified adaptive threshold methodology. Identified CAPs were reviewed and signals that might have been erroneously captured by the adaptive threshold were manually removed. We ignored all areas of signal saturation, as well as signals corresponding to cardiac and respiratory components. Following this signal processing, information regarding rate and temporal coding patterns were extracted and further analyzed using OriginPro software (version 8, OriginLab). Neurograms were defined as the first interval of time in which the CAP frequency was $>3 \times$ baseline level. TNF and IL-1 $\beta$ neurograms were extracted and subjected to fast Fourier transform for power spectral density (PSD) analysis (frequency resolution of $0.98 \mathrm{~Hz}$, using a Hanning window). Within the frequency domain, notch filters were applied $(60 \pm 10$ and $120 \pm$ $10 \mathrm{~Hz}$ ) to minimize the contribution of electrical noise along with its dominant harmonic. Data were linearly interpolated between the notch-filtered intervals. The areas under the PSDs (0-400 Hz range) were calculated for each cytokine response.

\section{Nodose Ganglion Cultures}

The nodose ganglia harvest protocol was adapted from previously published reports (27). Briefly, nodose ganglia were excised from euthanized mice under optical magnification. Connective tissue 

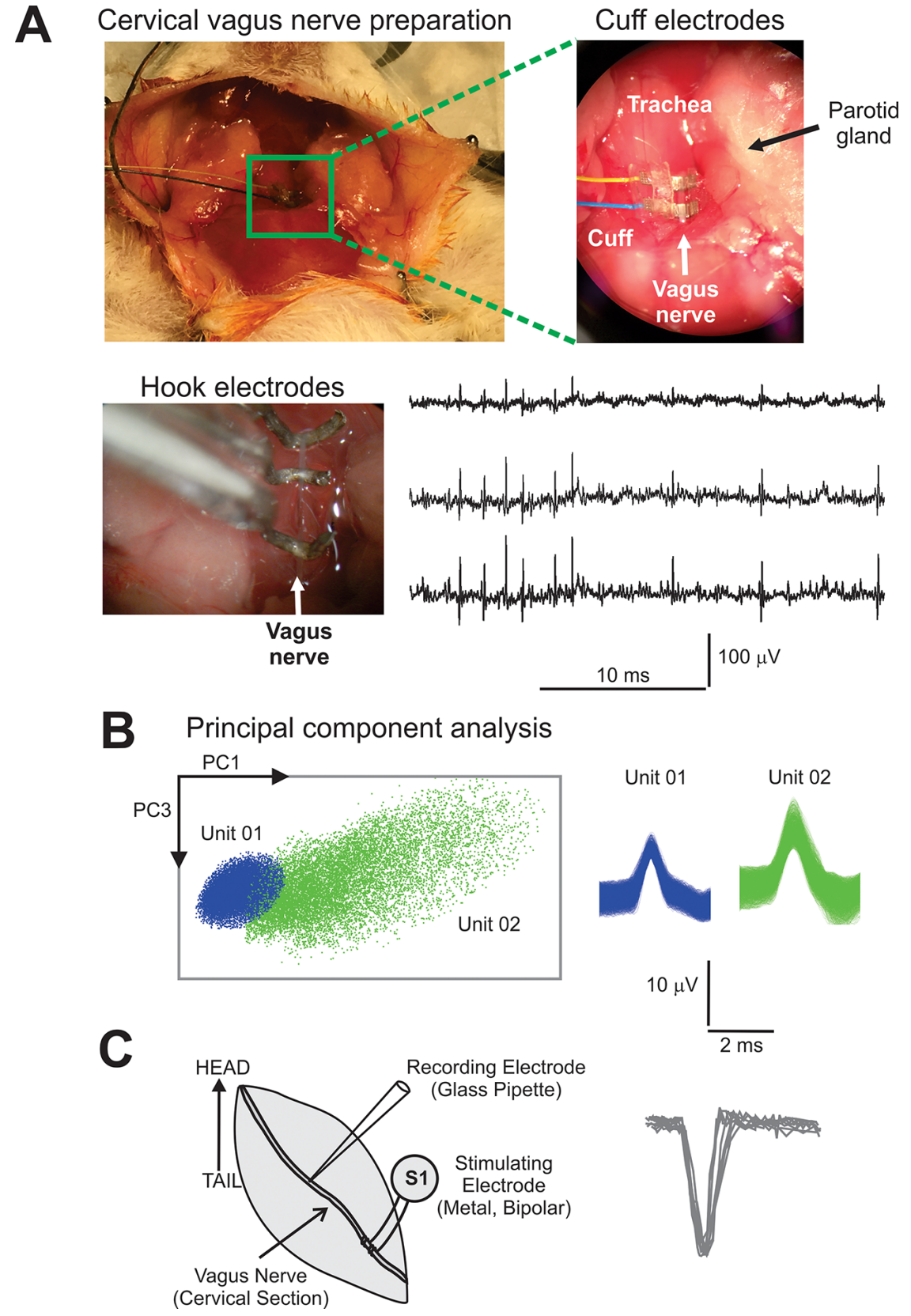

(Cervical Section)

Figure 1. Electrophysiological recording system for the cervical vagus nerve. (A) (Top left) Surgical procedure used to place the cuff electrodes in the cervical vagus nerve of an anesthetized BALB/c mouse. (Top right) Enlargement indicating the cuff electrodes, the vagus nerve and neighboring structures. (Bottom left) Lab-made hook electrodes used to record compound action potentials (CAPs) from the vagus nerve. (Bottom right) Representative recording of the neural signals by three adjacent hook electrodes. (B) (Left) By using standard template matching and spike-sorting algorithms relying on principal component analysis, unit spikes are discriminated in the vagus nerve recordings. Most large-amplitude waveforms correspond to CAPs, but spikes in the 5-10 $\mu \mathrm{V}$ amplitude range are likely carried by single fibers. (Right) Putative single-fiber units identified by spike sorting. (C) (Left) Diagram of the axon-attached recordings triggered by a brief stimulating pulse (S1). (Right) Examples of superimposed single-fiber action potentials (shown as negative waves). Same scale as in B. was digested using collagenase and dispase $(100 \mathrm{mg} / \mathrm{mL})$ for $1 \mathrm{~h}$ at $37^{\circ} \mathrm{C}$. Cells were washed with Neurobasal medium, then triturated prior to plating in poly-D-lysine and laminin-coated tissue culture wells. Cultures were maintained in Neurobasal medium supplemented with B27, GlutaMAX, NGF (25 ng/mL), and antibiotic for $4 \mathrm{~d}$ prior to use for immunocytochemistry or intracellular calcium measurements.

\section{Intracellular Calcium Measurements}

Nodose ganglia were washed with Hank's buffered salt solution and loaded with the calcium-sensitive Fluo-4 NW with pluronic acid F-127 (Molecular Probes) for $60 \mathrm{~min}$, then mounted on the stage of an Axiovert 200M inverted fluorescence microscope (Carl Zeiss Microscopy). The sample was illuminated every $10 \mathrm{~s}$ with light from a mercury lamp passing through an excitation filter $(470 \mathrm{~nm} \pm 40)$ before being directed at the cells via a $495 \mathrm{~nm}$ dichroic mirror. A cooled CCD camera (AxioCam monochromatic, Carl Zeiss Microscopy) captured the emitted light after it was passed through a $525 \mathrm{~nm} \pm 50$ emission filter. The acquisition hardware was controlled by Axiovision 4.8 software (Carl Zeiss Microscopy). After being placed on the microscope stage, cells were washed once and baseline fluorescence recorded for $3 \mathrm{~min}$, at which time the neurons were treated with either TNF (100 ng/mL) or IL-1 $\beta$ (100 ng/mL). Intracellular fluorescence was monitored for an additional 10 min before concluding the experiment by treating with $55 \mathrm{mM} \mathrm{KCl}$ to depolarize the neurons. The acquired images were exported to ImageJ software for analysis. The mean fluorescence intensity values of the visualized cells were extracted for each time point, background subtracted, and normalized to their average baseline intensity. Individual neurons were identified by morphology and considered viable and appropriate for final statistical analysis if they exhibited an abrupt increase in intracellular calcium with $\mathrm{KCl}$ depolarization. A cell was defined as responsive to the cytokine if its fluorescence 
increased twofold during the first $5 \mathrm{~min}$ of treatment with cytokine. Each well was considered an individual experiment.

\section{Immunocytochemistry}

Neurons were fixed for $10 \mathrm{~min}$ in $4 \%$ paraformaldehyde and $30 \%$ sucrose solution (50:50) warmed to $37^{\circ} \mathrm{C}$, then washed with PBS containing $0.1 \%$ Triton- $X$ for $10 \mathrm{~min}$, followed by three rinses in PBS only. Neurons were blocked with $10 \%$ bovine serum albumin in PBS for $1 \mathrm{~h}$ at room temperature. After blocking, the cultures were incubated in primary antibodies for TNFR1 (clone HM104; Thermo Scientific) or IL1R (clone H-8; Santa Cruz Biotechnology) overnight at $4^{\circ} \mathrm{C}$. The cells were rinsed $3 \times$ in PBS and incubated in secondary antibodies overnight at $4^{\circ} \mathrm{C}$ prior to washing and mounting on slides. In control coverslips treated in the same manner except without primary antibodies, there was no immunofluorescence.

\section{Statistical Tests}

Data are presented as individual samples, mean \pm standard deviation (SD), and mean \pm standard error of the mean (SEM). The Shapiro-Wilk test was used to test for normality. Analysis of variance, Student $t$ test, Mann-Whitney test and Kolmogorov-Smirnov test were used to examine for statistical significance. $P$ values $<.05$ were considered significant.

All supplementary materials are available online at www.bioelecmed.org.

\section{RESULTS}

\section{Vagus Nerve Recordings Capture Neural Compound Action Potentials}

To investigate neural activity in the vagus nerve, we developed an electrophysiological recording system with either hook or cuff electrodes (Figure 1). The signals were digitized and subjected to high-pass filtering and post-acquisition smoothing algorithms (as described in Methods). The recorded signals showed periodic excursions from baseline that corresponded to the cardiac (amplitude:
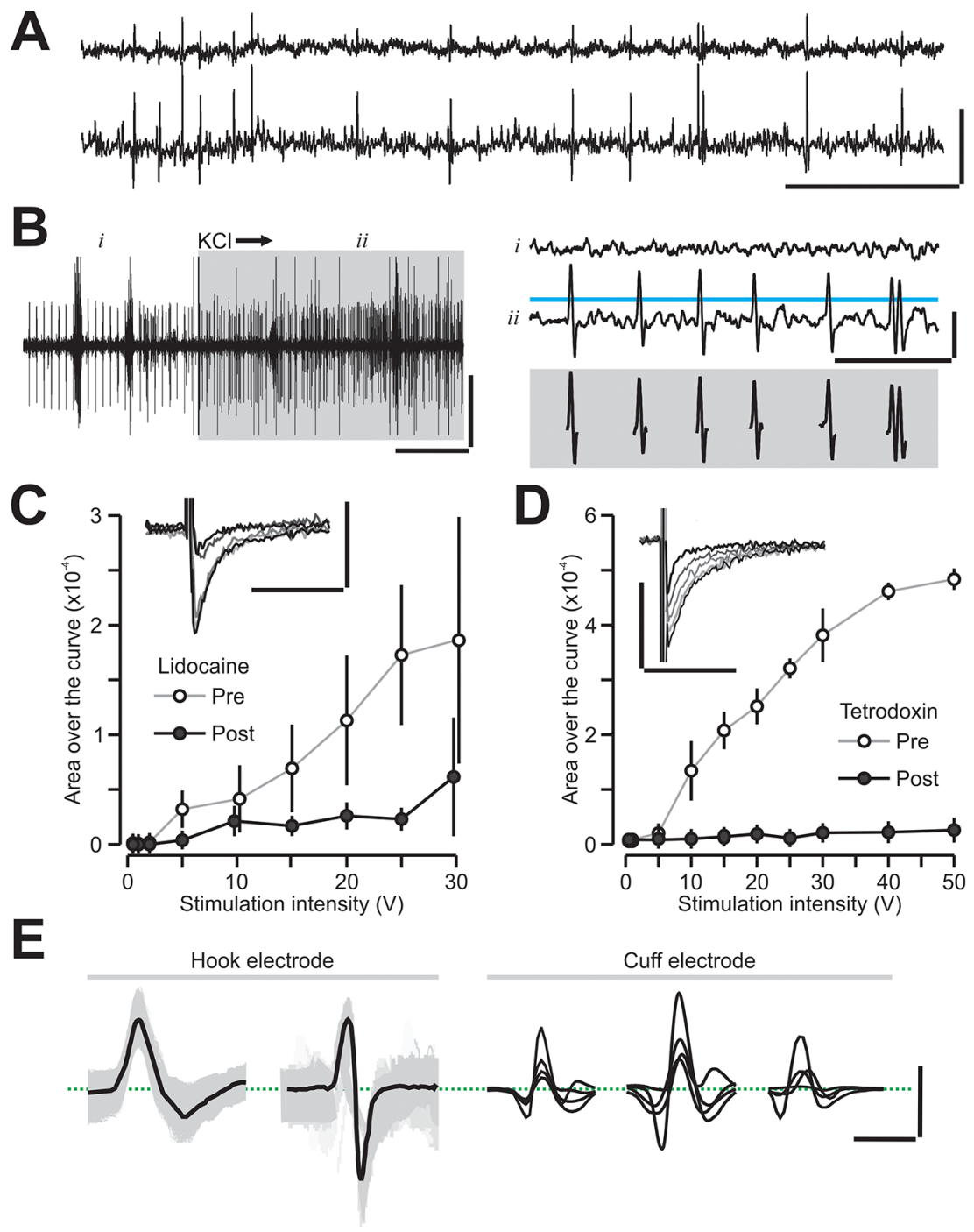

Figure 2. Recording compound action potentials in the vagus nerve. (A) Simultaneous recordings (two of three electrodes are shown) of spontaneous activity in the vagus nerve. (B) (Left) Vagus nerve excitation by $\mathrm{KCl}$ ( $4 \mathrm{mM}$, gray box) applied to the surgical field. (Top right) Period of quiescent activity before $\mathrm{KCl}$ (marked by $i$ ). (Middle) Period of intense spiking during $\mathrm{KCl}$ (marked by $\mathrm{ii}$ ). Blue line indicates the adaptive threshold used to detect compound action potentials (CAPs). (Bottom) Isolated CAPs are aligned with the trace above. (C) (Inset) Brief electrical pulses stimulate the vagus nerve and produce evoked CAPs. The graph shows the CAP area (mean \pm SD), which becomes larger with increasing stimulation intensity (pre circles). Lidocaine (2\%) largely blocks the evoked CAPs (post circles). (D) (Inset) Evoked CAPs previous to treatment with tetrodotoxin (100 $\mu \mathrm{M})$. The graph shows the CAP area (mean \pm SD), which is completely blocked by the drug. (E) Representative firing of single unit fibers obtained with hook and cuff electrodes. Scale bars $(x, y), A, 100 \mathrm{~ms}, 100 \mu \mathrm{V}$; B left, $1 \mathrm{sec}, 20 \mu \mathrm{V}$; B right, $10 \mathrm{~ms}, 20 \mu \mathrm{V} ; C, D, 2 \mathrm{~ms}$, $40 \mu \mathrm{V} ; \mathrm{E}, 2 \mathrm{~ms} .10 \mu \mathrm{V}$.

30-80 $\mu \mathrm{V}$, lasting 4-5 ms) and respiratory components (amplitude: $\sim 50 \mu \mathrm{V}$, lasting $\sim 120 \mathrm{~ms}$ ). Notably, contained within the signals were discrete, aperiodic spikes of variable amplitude that lasted $\sim 2 \mathrm{~ms}$ (Figure 2A). We employed an adaptive threshold technique to identify these fast spikes (Figure 2B) and surmised 
that they corresponded to compound action potentials (CAPs), which were generated by the near unison firing of multiple axons. To test whether the spikes indeed reflected neuronal activity, axonal activation and inhibition studies were performed. In the former, following baseline acquisition, $\mathrm{KCl}(4 \mathrm{mM})$ was applied to the surgical field near the vagus nerve so as to depolarize the fibers. This treatment produced a significant increase in neural activity (Figure 2B), with individual CAPs showing large amplitude and stereotypical shape (Figure 2B). By electrically stimulating the vagus preparation with brief pulses of increasing intensities, evoked CAPs of increasing amplitude were obtained (Figures 2C, D). Application of lidocaine, a potent anesthetic that blocks sodium channels, resulted in an almost total disappearance of the evoked CAPs (Figure 2C). Moreover, application of TTX, a highly specific blocker of axonal sodium channels, abrogated the evoked CAPs completely (Figure 2D), corroborating the neural nature of our nerve recordings. Remarkably, inhibition of the neural activity did not completely eliminate the cardiac and respiratory components, which were likely a composite of electrocardiac signals, electromyographic signals and motion artifacts. Although we focused on analyzing CAPs, occasionally we were able to discriminate putative unitary spikes that were likely carried by single fibers (Figures 1B, 2E). Cell-attached recordings from axons of the vagus nerve confirmed the detection of single-fiber action potentials (Figure 1C).

\section{Afferent Nature of the Vagus Neurograms}

We isolated and recorded the cervical vagus in anesthetized mice $(N=119)$ and used stringent inclusion/exclusion criteria (Figure 3A) to select experiments for further analysis $(N=83$ recordings lasting $>10 \mathrm{~min}$ ). Examination of the spontaneous baseline activity of the vagus showed minimal activation (Figure 4A) with an underlying mean CAP frequency of $5.61 \pm 0.74 \mathrm{~Hz}$ (mean $\pm \mathrm{SEM}, N=83$, Figure 3B). Traces were divided into $60-\mathrm{sec}$

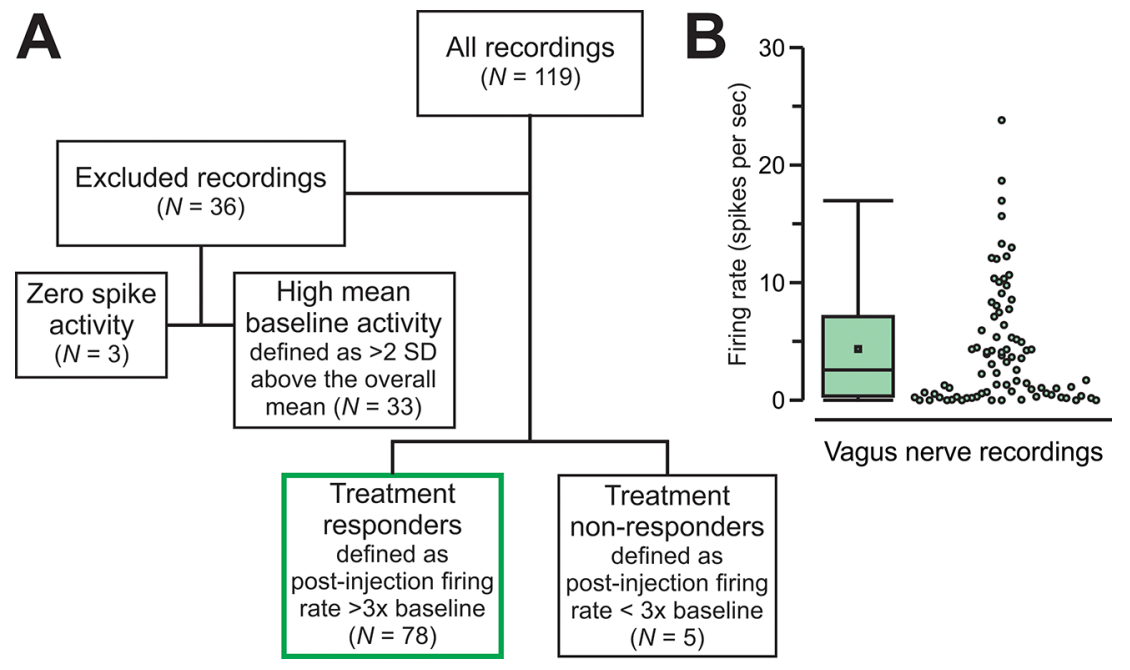

Figure 3. Criteria for selecting vagus nerve recordings for analysis. (A) The diagram shows the inclusion/exclusion criteria used to select experiments for further analysis. From a total of 119 cervical vagus recordings performed in anesthetized mice, 83 recordings lasting $>10$ min were selected. (B) The box plot shows the spontaneous activity of the vagus nerve during baseline recording for all the included experiments. Mean CAP frequency of $5.61 \pm 0.74 \mathrm{~Hz}$ (mean $\pm \mathrm{SEM}, \mathrm{N}=83$ ). Each dot represents a separate experiment.

events, which revealed long periods of complete quiescence $(N=85$ events with $\mathrm{CAP}$ frequency $=0$ ) that were punctuated by brief periods of strong neural activity. The CAP frequency distribution for nonsilent events was not normally distributed $(N=275, P=0, W=0.69$, Shapiro-Wilk test), with most events occurring at very low frequency and a few occurring at the high-frequency tail (Figure 4B). An initial test of our hypothesis that an organism's peripheral inflammatory status may represent a component of the vagus's sensory function was performed by applying TNF (50 $\mu \mathrm{g}$ in $200 \mu \mathrm{L}$ saline i.p.) while continuously recording vagus nerve activity. The resulting post-injection neurogram showed an acute increase in neural activity (Figure 4A), with a fraction of CAPs occurring at much higher frequency than baseline (Figure 4B). Administering trypsin-digested TNF, however, did not elicit an increase in CAP frequency (Supplementary Figure S1), strongly suggesting that the TNF neurogram was triggered specifically by the active cytokine and did not reflect spurious responses to the presence of exogenous protein.
The enhanced neurogram following TNF administration might reflect sensory signals, motor signals or both traveling through the vagus nerve. To directly test the signal directionality, we carried out proximal and distal vagotomies (relative to the brain) prior to administering TNF (Figure 4C). With a proximal cut, the afferent fibers tracking toward the recording electrodes from the visceral organs remained intact while efferent fibers no longer interfaced with the electrodes. The opposite was true with the distal vagotomy, in which the recording electrodes no longer accessed afferent fibers but the efferent fibers remained intact. For each experiment, CAPs were identified and the mean CAP frequencies were plotted across recordings (Figure 4D). To evaluate the responses, 10 min intervals immediately before and after TNF injection were considered. With proximal vagotomy, the CAP frequency showed a significant increase from baseline (pre, $5.8 \pm 2.8$; post, $39.5 \pm 9.1 \mathrm{~Hz}$; mean \pm SEM). In stark contrast, distal vagotomy resulted in a null enhancement (pre, 5.7 \pm 1.3 ; post, $2.9 \pm 1.5 \mathrm{~Hz}$; mean $\pm \mathrm{SEM}$ ). Comparing the individual post-injection 


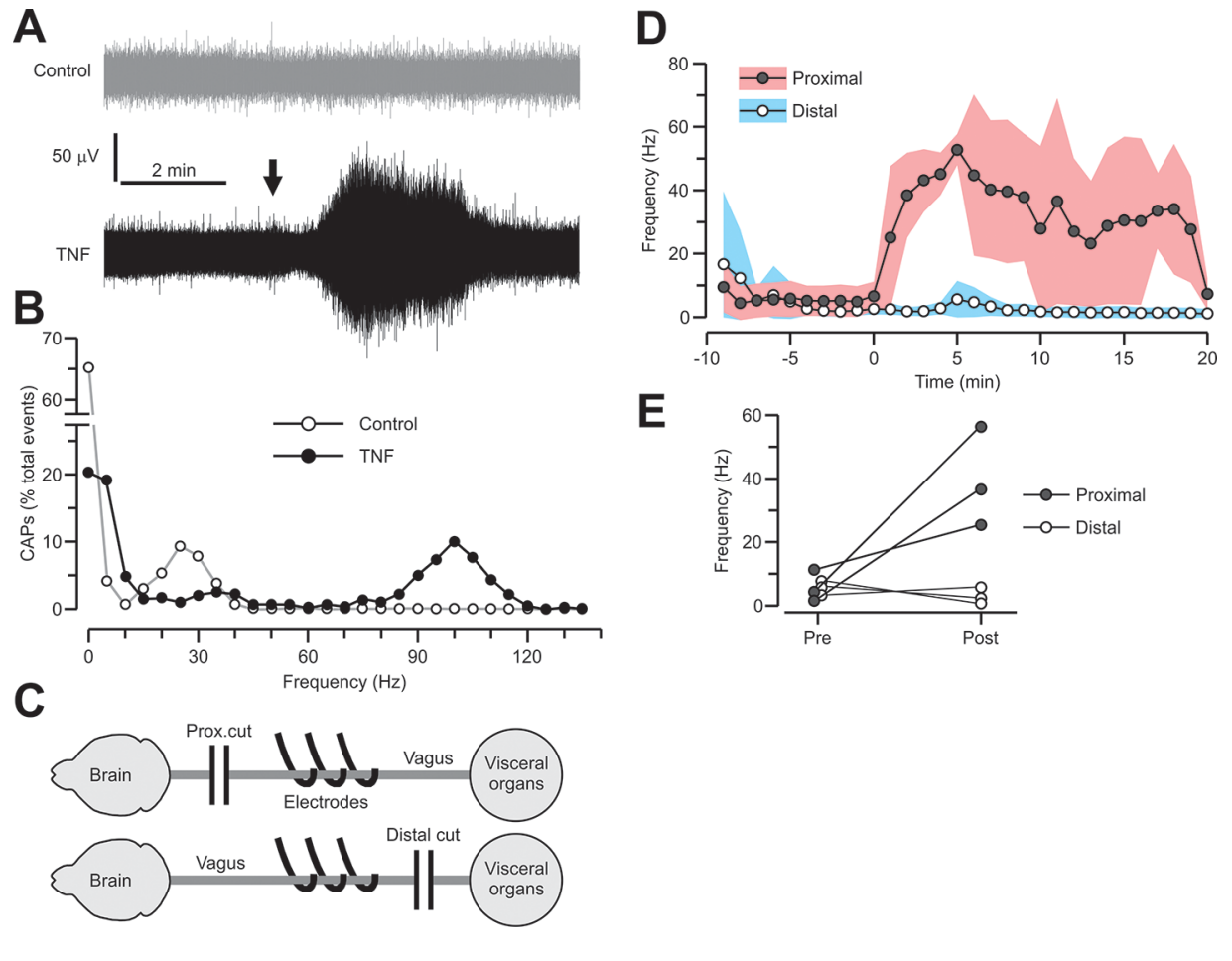

Figure 4. Afferent fibers of the vagus nerve carry TNF-induced neurograms. (A) (Top) Trace showing the spontaneous activity of the cervical vagus nerve. (Bottom) Trace showing the injection of TNF (dose of $50 \mu \mathrm{g}$, marked by line). (B) Graph depicting the frequency of CAP firing for the control and TNF-induced neurograms (shown in A). The 10-min period immediately after TNF injection is used to calculate CAP firing, as well as the equivalent period from the baseline. (C) Diagrams of the surgical vagotomies employed to test the direction of flow of the TNF-induced neurogram. A proximal (Prox) cut between the electrodes and the brain isolates the sensory component, while a distal cut isolates the efferent arm. (D) Graph showing the frequency of CAP firing in 60-s bins (mean \pm SEM, line \pm shaded area) starting $10 \mathrm{~min}$ prior to TNF injection (dose of $50 \mu \mathrm{g}$ ) at time zero. Data represent $N=3$ for each of proximal (filled circles) and distal (open circles) vagotomies. (E) Plot showing mean CAP frequencies for the 10-min periods before and right after TNF in individual mice. The distal cut completely abolishes the TNF effect, which is not affected by the proximal cut ( $P=.03$ for post values, $t$ test), indicating that afferent fibers are required.

CAP frequencies (Figure 4E) further demonstrated the significant difference between proximal and distal cuts $(P=.03$, $T=3.28, t$ test). These data strongly suggest that the first response recorded in the cervical vagus neurograms following cytokine administration includes and requires the sensory function of the vagus nerve.

\section{Vagus Neurograms Induced by Proinflammatory Cytokines}

We evaluated the neural activity associated with inflammatory stimuli by comparing the patterns of responses between TNF and IL-1 $\beta$. We used exogenous purified cytokines that were injected directly into the peritoneal cavity. Administration of vehicle $(200 \mu \mathrm{L}$ saline i.p.) did not induce any significant increase in vagus CAP activity compared with baseline (Figure 5A). Application of TNF (50 $\mu \mathrm{g}$ in $200 \mu \mathrm{L}$ saline i.p.) was followed by a notable increase in activity (Figure 5B). Indeed, comparing 10-min intervals immediately before and after TNF revealed a significantly higher mean CAP frequency after TNF (pre, $3.5 \pm 0.8$; post, $12.4 \pm 2.3$ $\mathrm{Hz} ; \mathrm{N}=21, P=.0006, T=4.0$, t test). The peak CAP frequency, defined as the maximum value within the interval, was also significantly elevated after TNF (pre, $38.9 \pm 9.7$; post, $71.4 \pm 10.9 \mathrm{~Hz}$; $N=21, P=.001, T=3.8, t$ test). Both the onset and termination of the activity envelopes were rapid, occurring on the order of seconds. Moreover, the period that followed the TNF-induced enhancement had a mean CAP frequency $(7.4 \pm 4.7 \mathrm{~Hz})$ that was similar to baseline activity.

Next, the vagus neurograms were evaluated in the context of IL-1 $\beta$ injection (350 ng per $\mathrm{kg}$ in $200 \mu \mathrm{L}$ saline i.p.). Interestingly, the peak response to IL- $1 \beta$ occurred earlier than that of TNF (Figure 5C). For this reason, we decided to use a 5 -min interval to evaluate the responses immediately before and after IL-1 $\beta$. The mean CAP frequency was significantly higher following the cytokine (pre, $6.2 \pm 2.5$; post, $26.1 \pm 8.3 \mathrm{~Hz}, N=10$, $P=.01, Z=2.5$, Mann-Whitney [MW] test), as was the peak CAP frequency (pre, $21.2 \pm$ 9.3; post, $80.9 \pm 14.7 \mathrm{~Hz}$; $N=10, P=.003, Z=3.0, \mathrm{MW}$ test). Following the enhanced IL-1 $\beta$ neurogram, the mean CAP frequency returned to baseline level $(4.4 \pm 1.9 \mathrm{~Hz})$. Importantly, the increased vagus activity that was triggered by cytokines did not reflect physical trauma or disruption to the peritoneal cavity and its viscera, because injection of vehicle alone $(200 \mu \mathrm{L}$ sterile saline i.p.) did not elicit a change in vagus activity (Figure 5A), as evidenced by the mean CAP frequency (pre, $5.5 \pm 0.92$; post, $5.3 \pm 1.1 \mathrm{~Hz} ; N=6$, $P=.9, Z=0.08$, MW test).

To investigate the dose dependency of the cytokine-induced neurograms, we applied increasing amounts of proinflammatory cytokines. We found that TNF (0.05-50 $\mu \mathrm{g}$ ) produced a dose-dependent increase in CAP activity during the 10-min interval immediately following TNF injection, with the exception of the $5-\mu \mathrm{g}$ dose (Figure 5D). Similarly, the number of CAPs dose-dependently increased in the 5 min following IL- $1 \beta$ administration when the IL- $1 \beta$ was titrated from 3.5 to $350 \mathrm{ng} / \mathrm{kg}$ (Figure 5E). The shorter time 

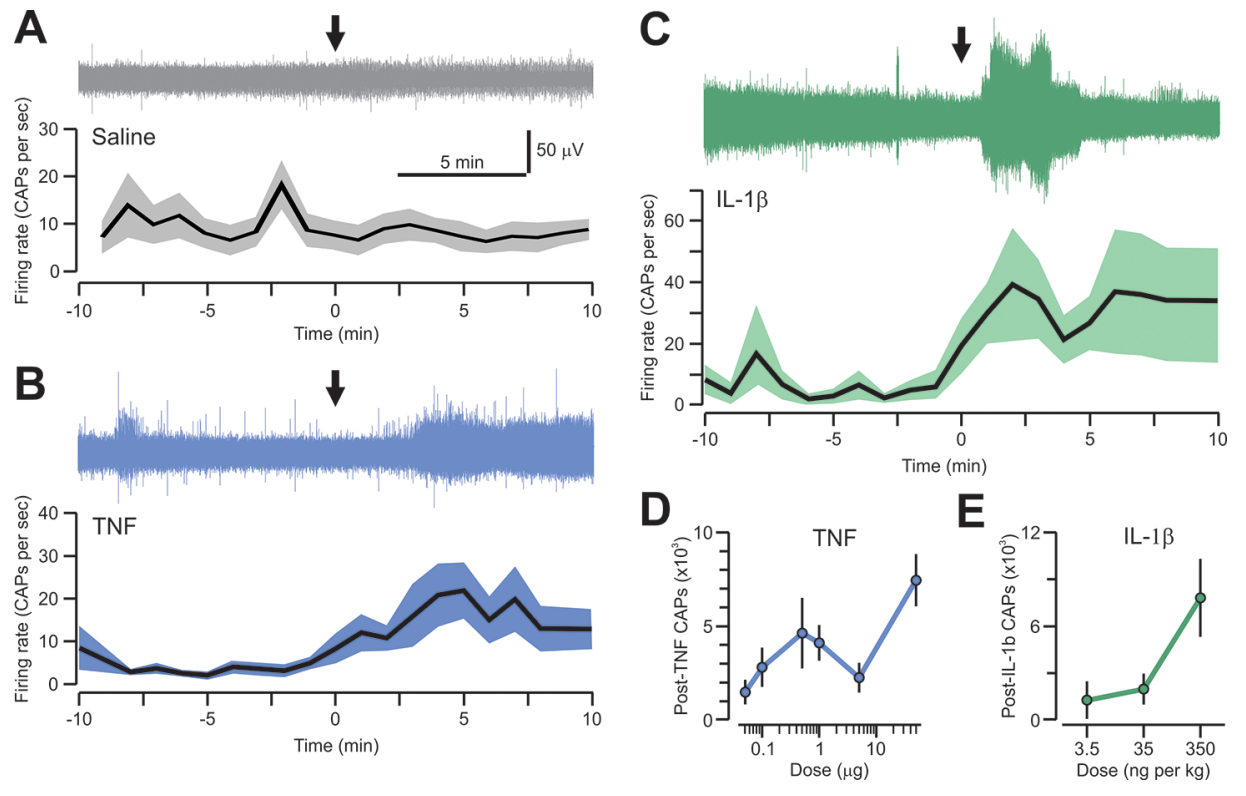

Figure 5. Time course for TNF- and IL-1 $\beta$-mediated neurograms. (A) (Top) Trace showing vagus nerve activity under vehicle ( $200 \mu \mathrm{L}$ sterile saline, marked by line), which is used as control. (Bottom) Graph describing the frequency of CAP firing (mean \pm SEM, gray area) for $N=6$ mice starting 10 min prior to saline injection. (B) (Top) Representative neurogram for TNF (dose of $50 \mu \mathrm{g}$, marked by line). (Bottom) Graph showing the mean frequency \pm SEM (blue area) for $N=21$ mice. (C) (Top) Representative neurogram for IL-1 $\beta$ (dose of 350 ng, marked by line). (Bottom) Graph showing mean frequency \pm SEM (green area) for $N=10$ mice. (D) Plot depicting the total CAP counts (mean \pm SEM) in the $10 \mathrm{~min}$ immediately after TNF administration at the indicated doses $(N=5-7$ mice per dose). (E) Plot showing total CAP counts in the 5 min following $\mathrm{IL}-1 \beta$ treatment at the indicated doses $(N=5-9$ mice per dose).

intervals used for IL-1 $\beta$ compared with TNF reflected the different temporal profiles of neurogram activation to IL-1 $\beta$ compared with TNF. In particular, the IL-1 $\beta$ response peaked earlier (150 sec post-injection) as compared with TNF (300 sec post-injection).

\section{Differences Between Neurograms Induced by TNF and IL-1 $\beta$}

Comparing the total CAPs (Figure 6A) for the 10-min post-injection period revealed differences between groups (saline, $2739.2 \pm 661.4, N=6$; TNF, 7444.6 \pm 1387.8, $N=21 ; \mathrm{IL}-1 \beta, 16893.4 \pm 6900.7, N=10$ ) that were significant (saline versus TNF, $P=.01, Z=2.4$; saline versus IL- $1 \beta, P=.01$, $Z=2.5$, MW test). However, there was no difference between the proinflammatory cytokines $(P=.07, T=1.9, t$ test). Next, we compared the temporal profiles of neurogram activation with TNF and IL-1 $\beta$ administration by defining the beginning of the response as the time at which the CAP frequency increased to $3 \times$ the preinjection rate, and the response termination as the time when the frequency decreased below the same value. Using these definitions, we analyzed the latency, duration and mean CAP frequency for the TNF- and IL-1 $\beta$ specific responses. The latencies for the neurograms induced by TNF $(N=21)$ and IL-1 $\beta(N=10)$ were not significantly different (Figure 6B; TNF, 150.6 \pm 33.1; IL-1 $\beta, 77.1 \pm 31.6 \mathrm{sec} ; P=.16, T=1.4, t$ test). Moreover, the response duration (TNF, 286.9.8 \pm 40.9; IL-1 $\beta, 406.8 \pm 59.5$ sec; $P=.10, T=1.7, t$ test) and peak CAP rate $(\mathrm{TNF}, 58.9 \pm 8.1 ; \mathrm{IL}-1 \beta, 72.8 \pm 15.8$ $\mathrm{Hz} ; P=.39, \mathrm{~T}=0.87, t$ test) were not significantly different.

\section{Spectral Power of the Neurograms Induced by TNF and IL- $1 \beta$}

Given that TNF and IL-1 $\beta$ elicited similar, but not identical, enhancements in the vagus nerve neurograms and that these signals involved afferent fibers, we sought to examine whether the vagus nerve is capable of sending distinct signals about TNF and IL-1 $\beta$ to the CNS. To investigate this possibility, the PSDs of the TNF and IL- $1 \beta$ responses were analyzed (Figure 6C) using unfiltered neurogram recordings. As described above, the response was defined as the first interval of time at which the CAP frequency resided at $3 \times$ the baseline level. Next, each filtered PSD was integrated $(0-400 \mathrm{~Hz})$ to compare the individual areas of the TNF $(N=14)$ and IL-1 $\beta(N=7)$ responses (Figure 6D). Statistical analysis revealed a significant difference between TNF and IL- $1 \beta$ groups $(P=.04, Z=2.05$, MW test). Together, the differences in the response temporal characteristics and frequency domain power analyses indeed demonstrated selective cytokine neural sensory signaling through the vagus nerve.

\section{Receptor Requirement for the Neurograms Induced by TNF and IL- $1 \beta$}

It has previously been demonstrated that TNF trimers can form receptorindependent transmembrane channels (12). In this case, administration of TNF could in theory directly activate neurons without engaging its receptor. To test whether the cytokine-induced responses are cytokine receptor-dependent, the vagus nerve neurograms from receptor $\mathrm{KO}$ mice were recorded. We found that TNF injection in double $\mathrm{KO}$ mice for TNFR1 and TNFR2 (TNF-R1/2KO) did not lead to a change in the neurogram, whereas IL-1 $\beta$ injection in these mice elicited neurogram enhancement (Figure 7A; $P=.04, Z=1.97$, MW test) implicating a ligand-receptor interaction in the observed TNF response. Next, the requirement for the IL- $1 \beta$ receptor (IL1R) in the IL-1 $\beta$-induced neurogram 
A

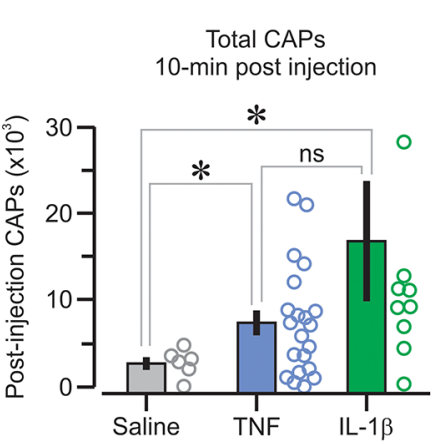

C

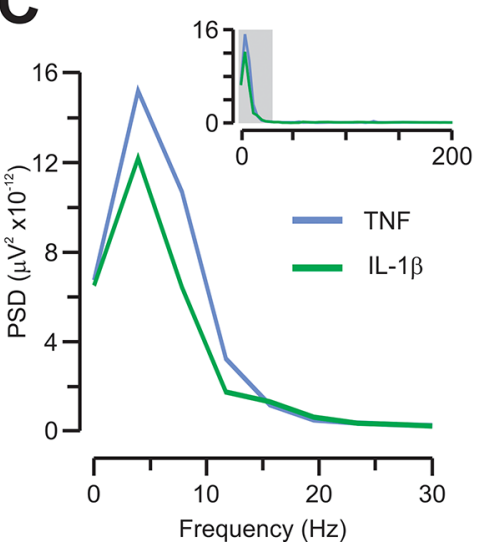

B

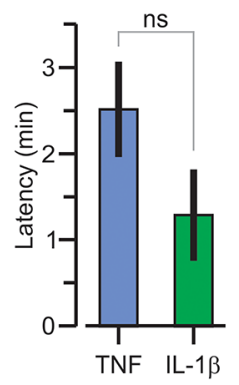

D

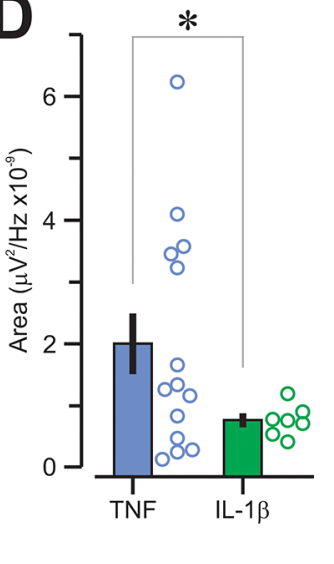

Figure 6. Time domain and spectral analysis of TNF- and IL-1 $\beta$-mediated neurograms. (A) Plot showing the total CAP counts in the 10-min period immediately after cytokine administration (saline, $200 \mu \mathrm{L}$ per mouse, $N=6$; TNF, $50 \mu \mathrm{g}$ per mouse, $N=21$; IL-1 $\beta$, $350 \mathrm{ng} / \mathrm{kg}, N=10$ ). Values between the saline control and each cytokine are statistically significant ( ${ }^{*}, P<.05, \mathrm{MW}$ test), but the cytokines are not significantly different from each other ( $n s, P=.07, t$ test). (B) Graph depicting the latency (mean \pm SEM) for the TNF and IL-1 $\beta$ responses, with no significant difference between values $(P=.16, t$ test). (C) Power spectral densities (PSDs) for the TNF (blue) and IL-1 $\beta$ (green) responses in the 0-30 Hz range for the unfiltered neurogram recordings. (Inset) PSD for the 0-200 Hz range, with the gray rectangle indicating the expanded plot. (D) The areas under the PSDs ( $0-400 \mathrm{~Hz}$ range) were calculated for TNF and IL-1 $\beta$. The calculated area for each response is shown for TNF (blue) and IL-1 $\beta$ (green). Responses are statistically different ( ${ }^{*}, P<.05, \mathrm{MW}$ test, $N=7$ for each group), suggesting a potential biological substrate for cytokine discrimination within the CNS.

activation was tested in IL1R knockout (IL1RKO) mice. Exogenous IL-1 $\beta$ did not stimulate the cervical vagus nerve in IL1RKO mice, while TNF significantly increased neural activity (Figure 7B; $P=.045, Z=1.98$, MW test). In all $\mathrm{KO}$ mouse experiments in which no clear response to either TNF or IL1 $\beta$ was observed, $\mathrm{KCl}$ (4 mM) was applied to the vagus nerve at the termination of the experiments to ensure nerve viability.

\section{Sensory Vagus Neurons from Nodose Ganglion Express Cytokine Receptors That Respond to Exogenous Cytokines}

We next sought mechanistic insight into how TNF and IL-1 $\beta$ enhance vagus nerve activity. In our in vivo preparation, proinflammatory cytokines may directly activate sensory vagus nerve fibers within the peritoneum. Alternatively, intermediate populations of receptor-expressing somatic cells may be required to sense the cytokine and, in turn, stimulate the neurons. Several studies have demonstrated the presence of functional cytokine receptors within neuronal populations such that activation of these receptors is capable of modulating neuronal excitability (9-11). A recent report has similarly shown that bacterial products can directly activate a specific population of sensory neurons (13). Accordingly, we hypothesized that the cytokines directly engage their respective receptors on vagus nerve afferents, leading to increased nerve activity. To address this possibility, we cultured nodose ganglia neurons from adult mice and analyzed cytokine receptor expression. The cultures contained only neurons and were devoid of immune or other supportive cells. By immunocytochemistry, nodose ganglia neurons from wild type (WT) animals showed surface expression of the TNF and IL-1 $\beta$ receptors (Figure 7C), supporting the notion that the cytokines may directly bind receptors on the neurons.

We next evaluated cytokine-dependent nodose ganglia neuronal activation by intracellular calcium measurements with fluorescence microscopy. Cultured neurons were loaded with the calciumsensitive dye Fluo-4 and imaged before and after the addition of either TNF (100 ng/mL) or IL-1 $\beta$ (100 ng/mL). Both cytokines elicited increased intracellular calcium in subpopulations of neurons harvested from WT but not TNFR1/2RKO or IL1RKO animals, respectively (Figure 7D; TNF1/2RKO versus $W T, P=.002, Z=3.03$; IL1RKO versus $W T, P=.01, Z=2.5, \mathrm{MW}$ test). Together, these data support a direct mechanism of vagus nerve activation through cytokine-receptor engagement on the neuron membrane.

\section{DISCUSSION}

The work presented here adds to the rapidly expanding literature describing the interface between neuroscience and immunology, delineating for the first time the afferent arc of the inflammatory reflex. We established methodologies for recording cytokine-induced CAPs in the cervical vagus nerve. By electrically 

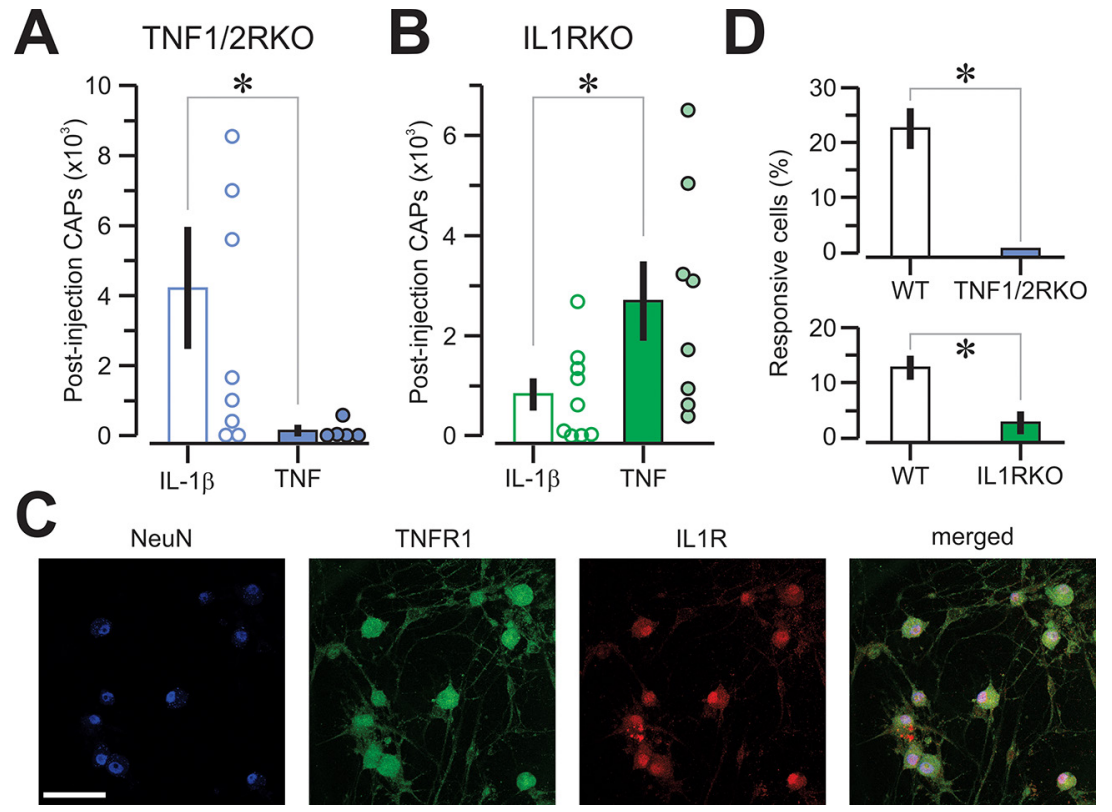

Figure 7. Genetic ablation of cytokine-mediated neurograms. (A, B) Plots showing the total CAP count in the 5-min period immediately after IL-1 $\beta$ or TNF administration in $\mathrm{KO}$ mice. (A) In TNFR1/2KO mice, responses for IL-1 $\beta(N=8)$ and TNF $(N=5)$ are significantly different ( ${ }^{*}, P<.05$, MW test). (B) In ILIRKO mice, CAPs for IL-1 $\beta(N=8)$ and TNF $(N=8)$ are also statistically significant ${ }^{*}, P<.05, \mathrm{MW}$ test). (C) Cultured nodose ganglia are stained for NeuN (left panel) to identify all cultured neurons, antibody for TNFR1 (green panel) and antibody for ILIR (red panel); merged signals are shown in the right panel. Scale bar, $50 \mu \mathrm{m}$. (D) Responsive neurons display $a \geq$ two-fold increase in Fluo-4 fluorescence in response to $100 \mathrm{ng} / \mathrm{mL}$ cytokine. (Top) Plot of neurons that respond to TNF in WT $(N=7)$ and TNFR1/2KO $(N=7)$ mice. (Bottom) Neurons that respond to IL-1 $\beta$ in WT $(N=5)$ and IL 1RKO $(N=4)$ groups; ${ }^{*}, P<.05$, MW test.

stimulating or suppressing the vagus with the sodium channel blockers lidocaine and TTX, we confirmed that the recorded activity is a function of neuronal response. Interestingly, the cell bodies of nodose sensory neurons contain Nav1.8, a TTX-resistant sodium channel (14-18), but the dose of TTX $(100 \mu \mathrm{M})$ we used leads to complete suppression of vagus nerve activity, in accordance with previous reports (14-18) and implying that the axons running through the cervical vagus predominantly express TTXsensitive sodium channels. Furthermore, using surgical vagotomies, we verified the postulate that afferent fibers of the vagus nerve can function as cytokine sensors that relay information to the CNS (6-8). Moreover, analysis of neurograms for TNF and IL-1 $\beta$ reveal selective, specific, afferent signaling of the vagus nerve in response to proinflammatory cytokines.

To study the receptor dependency of the sensory component of the inflammatory reflex, we used receptor $\mathrm{KO}$ mice. The absence of neurogram enhancement in response to TNF or IL-1 $\beta$ in the respective receptor $\mathrm{KO}$ animals implicates the cytokine receptor in cytokine-induced activation of the vagus nerve. Specifically, the cytokine-receptor interactions mediate the neurogram response. At the cellular level, these finding were corroborated in vitro by direct activation of a subset of cultured nodose ganglia neurons by exogenous cytokines. Consistent with this finding, our immunofluorescence studies demonstrate that vagus sensory neurons express TNF and IL-1 $\beta$ receptors. Together, our results support a mechanism of vagus nerve activation by cytokines. Whether this occurs in vivo remains speculative. Conditional receptor $\mathrm{KO}$ mice would be useful to address whether cytokines activate the neurons directly in vivo, or whether they mediate their effect through an intermediate player.

Remarkably, the differences between TNF and IL-1 $\beta$ suggest the intriguing possibility that the CNS might be able to discriminate among a diverse set of inflammatory mediators. This notion has a strong teleological basis, because the CNS receives a continuous sensory flow of information pertaining to the internal body environment, of which the signals that relay systemic inflammation must constitute a key element for an animal's homeostasis and survival. With the rapid delivery of signals specific to peripheral inflammatory (and immune) status, an organism would be better able to initiate appropriate physiological and behavioral responses to immunological and environmental challenges. Similarly, the vagus nerve sensory fibers may serve as an early monitoring system against invading microorganisms. This notion is consistent with reports of sensory neuron activation by bacterial products (13). Future work directed at generating a compendium of neural recordings in the context of endogenous and exogenous inflammatory stimuli will define the full extent to which the vagus nerve monitors the body's immunological status.

In addition to the observed temporal differences in neurogram enhancement between TNF and IL-1 $\beta$, the spectral densities of the individual responses (Figures 6C, D) provide further evidence for the existence of discriminating features between cytokine-elicited vagus nerve activities that could be interpreted by CNS centers, such as the nucleus tractus solitarius. There is ample evidence that many cortical and subcortical structures within the CNS can distinguish the spectral characteristics of signals and use them in processes as diverse as memory encoding, decision making and switching between sleep states (19-21). We propose that either alone or in combination, the 
observed differences in time and frequency domain metrics may represent the biological substrate for discrimination of peripheral cytokines by the CNS. While the specific information currency by which the brain differentially receives input about proinflammatory cytokines remains elusive, ongoing work in the development of more sensitive peripheral nerve recording systems, along with data science methodology, will likely lead to more refined definitions of cytokine-specific neural signatures through which the CNS monitors peripheral inflammation states.

The interface between neuroscience, immunology and clinical medicine is increasingly moving to the fore, especially since the use of electrical devices as therapeutic agents for disease becomes a reality and shapes the emerging field of bioelectronics medicine (22-24). For instance, a recent clinical trial that used vagus nerve stimulation to treat rheumatoid arthritis proved successful $(25,26)$. This approach represents the first step in the development of closed-loop bioelectric therapeutics. At present, electrical stimulation therapies involve preset, empirically derived stimulation paradigms. Stimulation protocols that are able to read, interpret and respond to a patient's active state of disease would afford a greater degree of therapeutic benefit by delivering patient-specific electric doses. An understanding of the sensory code of these neural reflexes is required to develop such a closed-loop system.

\section{CONCLUSION}

The interface between neuroscience, immunology and clinical medicine is increasingly moving to the fore. In this context, the vagus (tenth cranial) nerve plays a pivotal role as it connects the visceral organs with brain centers that respond to changes in the physiological and immunological status of the organism. In this study, we show that two pro-inflammatory cytokines (TNF and IL-1 $\beta$ ) produce significant enhancements of neural activity in the vagus nerve. These results provide mechanistic insight into the immunological sensory neural code.

\section{ACKNOWLEDGMENTS}

We thank Thomas Faust and Stephen Frattini for their help in pilot studies. We thank Yaakov Levine for suggestions about hook electrodes, and Jianhua Li for providing purified recombinant TNF. BES is supported by a Clinician Investigator Program fellowship from the Ministry of Health (Canada), the Joseph M. West Memorial Fund and the Javenthey Soobbiah Scholarship. PTH acknowledges National Institutes of Health/National Institute of Allergy and Infectious Diseases funding (grant 5P01-AI073693). This study was completed with grant support from the Defense Advanced Research Projects Agency (W911NF-09-1-0125) to KJT.

\section{DISCLOSURE}

The authors declare that they have no competing interests as defined by Bioelectronic Medicine, or other interests that might be perceived to influence the results and discussion reported in this paper.

\section{REFERENCES}

1. Tracey KJ. (2002) The inflammatory reflex. Nature. 420:853-59.

2. Rosas-Ballina M, Olofsson PS, Ochani M, et al. (2011) Acetylcholine-synthesizing T cells relay neural signals in a vagus nerve circuit. Science. 334:98-101.

3. Reardon C, Duncan GS, Brüstle A, et al. (2013) Lymphocyte-derived ACh regulates local innate but not adaptive immunity. Proc. Natl. Acad. Sci. USA. 110:1410-15.

4. Hansen MK, O'Connor KA, Goehler LE, Watkins LR, Maier SF. (2001) The contribution of the vagus nerve in interleukin-1beta-induced fever is dependent on dose. Am. J. Physiol. Regul. Integr. Comp. Physiol. 280:R929-34.

5. Watkins LR, Goehler LE, Relton JK, et al. (1995) Blockade of interleukin-1 induced hyperthermia by subdiaphragmatic vagotomy: evidence for vagal mediation of immune-brain communication. Neurosci. Lett. 183:27-31.

6. Niijima A, Hori T, Katafuchi T, Ichijo T. (1995) The effect of interleukin-1 beta on the efferent activity of the vagus nerve to the thymus. J. Auton. Nerv. Syst. 54:137-44.

7. Niijima A. (1996) The afferent discharges from sensors for interleukin 1 beta in the hepatoportal system in the anesthetized rat. J. Auton. Nerv. Syst. 61:287-91

8. Niijima A. (1992) Electrophysiological study on the vagal innervation of the adrenal gland in the rat. J. Auton. Nerv. Syst. 41:87-92.
9. Czeschik JC, Hagenacker T, Schäfers M, Büsselberg D. (2008) TNF-alpha differentially modulates ion channels of nociceptive neurons. Neurosci. Lett. 434:293-98.

10. Hagenacker T, Czeschik JC, Schäfers M, Büsselberg D. (2010) Sensitization of voltage activated calcium channel currents for capsaicin in nociceptive neurons by tumor-necrosis-factoralpha. Brain Res. Bull. 81:157-63.

11. Binshtok AM, Wang H, Zimmermann K, et al. (2008) Nociceptors are interleukin-1beta sensors. J. Neurosci. 28:14062-73.

12. Kagan BL, Baldwin RL, Munoz D, Wisnieski BJ. (1992) Formation of ion-permeable channels by tumor necrosis factor-alpha. Science. 255:1427-30.

13. Chiu IM, Heesters BA, Ghasemlou N, et al. (2013) Bacteria activate sensory neurons that modulate pain and inflammation. Nature. 501:52-57.

14. Muroi Y, Ru F, Kollarik M, et al. (2011) Selective silencing of NaV1.7 decreases excitability and conduction in vagal sensory neurons. J Physiol. 589:5663-76

15. Docherty RJ, Charlesworth G, Farrag K, Bhattacharjee A, Costa S. (2005) The use of the rat isolated vagus nerve for functional measurements of the effect of drugs in vitro. J. Pharmacol. Toxicol. Methods. 51:235-42.

16. Farrag KJ, Costa SK, Docherty RJ. (2002) Differential sensitivity to tetrodotoxin and lack of effect of prostaglandin E2 on the pharmacology and physiology of propagated action potentials. Br. J. Pharmacol. 135:1449-56.

17. Akopian AN, Sivilotti L, Wood JN. (1996) A tetrodotoxin-resistant voltage-gated sodium channel expressed by sensory neurons. Nature. 379:257-62.

18. Tate S, Benn S, Hick C, et al. (1998) Two sodium channels contribute to the TTX-R sodium current in primary sensory neurons. Nat. Neurosci. 1:653-55.

19. Chang EH, Huerta PT. (2012) Neurophysiological correlates of object recognition in the dorsal subiculum. Front. Behav. Neurosci. 6:46.

20. Nácher V, Ledberg A, Deco G, Romo R. (2013) Coherent delta-band oscillations between cortical areas correlate with decision making. Proc. Natl. Acad. Sci. USA. 110:15085-90.

21. Kopell N, Kramer MA, Malerba P, Whittington MA (2010) Are different rhythms good for different functions? Front. Hum. Neurosci. 4:187.

22. Famm K, Litt B, Tracey KJ, Boyden E, Slaoui M. (2013) Drug discovery: a jump-start for electroceuticals. Nature. 496:159-61.

23. Birmingham K, Gradinaru V, Anikeeva P, et al. (2014) Bioelectronic medicines: a research roadmap. Nat. Rev. Drug. Discov. 13:399-400.

24. Huerta PT, Olofsson PS. (2016) Bioelectronic medicine: Harnessing the electric patterns of neurons for therapy. Technical workshop W06 at the 10th Forum of Neuroscience, Copenhagen, Denmark. FENS; c.2016 [updated 2016 Jun 30; cited 2016 Dec 8]. Available from: http:/ / forum2016.fens. org/forum-programme/preliminary-programme/ 
25. Koopman FA, Schuurman PR, Vervoordeldonk MJ, Tak PP. (2014) Vagus nerve stimulation: a new bioelectronics approach to treat rheumatoid arthritis? Best Pract. Res. Clin. Rheumatol. 28:625-35.

26. Koopman FA, Chavan SS, Miljko S, et al. (2016)

Vagus nerve stimulation inhibits cytokine production and attenuates disease severity in rheumatoid arthritis. Proc. Natl. Acad. Sci. USA. 113:8284-89.

27. Simasko SM, Wiens J, Karpiel A, Covasa M, Ritter RC. (2002) Cholecystokinin increases cytosolic calcium in a subpopulation of cultured vagal afferent neurons. Am. J. Physiol. Regul. Integr. Comp. Physiol. 283:R1303-13.

Cite this article as: Steinberg BE, et al. (2016) Cytokine-specific neurograms in the sensory vagus nerve. Bioelectron. Med. 3:7-17. 